\title{
Establishment and Evaluation of Isoproterenol Induced Chronic Heart Failure and Cardiac Remodeling Model in Rats: An Experimental Study
}

\author{
Dan Huang ${ }^{1}$, Jianting Ke ${ }^{1}$, Anzhu Zhao², Zhisheng Yang ${ }^{2}$, Diefei Liang ${ }^{2}$, Jing Pan ${ }^{2}$ \\ Mao Liu ${ }^{1}$, Jian Chen ${ }^{1, *}$ \\ ${ }^{1}$ Department of Cardiology, Fifth Affiliated Hospital of Sun Yat-sen University, Zhuhai, P.R. China \\ ${ }^{2}$ Department of Clinical Medicine, Zhongshan School of Medicine, Sun Yat-sen University, Guangzhou, P.R. China \\ *Corresponding Author: chenjn@mail.sysu.edu.cn
}

Copyright $(\underset{C}{ } 2014$ Horizon Research Publishing All rights reserved.

\begin{abstract}
Objective: To assess the efficacy of isoproterenol (ISO) in the establishment of chronic heart failure (CHF) and cardiac remodeling model in rats. Methods: Twelve Wistar rats were randomly divided into two groups: control group $(n=3)$ and model group $(n=9)$. Rats in model group were hypodermic injected with ISO $5 \mathrm{mg} / \mathrm{kg} / \mathrm{d}$ for 10 days. Ultrasonic cardiogram, HE staining, immunohistochemistry of collagen I and Masson staining were performed to evaluate the cardiac function and myocardial fibrosis. Besides, ventricular mass/body mass ratio, collagen volume fraction $(\mathrm{CVF})$, perivascular collagen area (PVCA), hydroxyproline (HYP) concentration and relative expression of transforming growth factor $\beta 1$ (TGF- $\beta 1$ ) mRNA were also detected. Results: Compared to the controls, rats in model group had a marked enlargement of cardiac dilatation and reduction of ejection fraction (57.00 $\pm 3.61 \%$ vs. $44.67 \pm 3.06 \%, \quad \mathrm{P}=0.011)$. Ventricular mass/body mass ratio ( $3.60 \pm 0.31$ vs. $4.88 \pm 0.34, \mathrm{P}=0.020)$, CVF $(5.65 \pm 0.68 \%$ vs. $27.62 \pm 4.89 \%, \quad \mathrm{P}=0.020)$, PVCA $(11.22 \pm 3.40 \%$ vs. $28.50 \pm 4.52 \%, \mathrm{p}=0.001)$ and HYP level $(0.24 \pm 0.08 \mu \mathrm{g} / \mathrm{mg}$ wet weight vs. $0.62 \pm 0.11 \mu \mathrm{g} / \mathrm{mg}$ wet weight, $\mathrm{P}=0.001$ ) were significantly increased in model group. Remarkably cardiac fibrosis were also observed in the Masson staining and immunohistochemistry. The expression of TGF- $\beta 1$ mRNA was increased significantly in the model group $(\mathrm{P}=0.003)$. The mortality rate in the model group was $33.3 \%$ during the whole procedure. Conclusion: ISO can successfully induce a $\mathrm{CHF}$ and cardiac remodeling rat model with a low mortality rate.
\end{abstract}

Keywords Isoproterenol, Heart Failure, Cardiac Remodeling, Myocardial Fibrosis

\section{Introduction}

Chronic heart failure (CHF) is a complicated clinical syndrome with impaired ventricular filling and ejection function caused by the abnormality of structure or function. $\mathrm{CHF}$ is one of the most important cardiovascular diseases [1]. Therefore, the study on CHF and cardiac remodeling is constantly the focus of cardiovascular territory [2]. The methods for establishment of CHF and cardiac remodeling rat model include coronary artery ligation or embolization, high capacity load, high pressure load, drug-induced method and viral infection method. Drug-induced method is preferred because of easy operation and low cost. Previous study [3] suggests large dose of isoproterenol (ISO) could cause myocardial impairment in rats, which induced CHF and cardiac remodeling. However, there is relatively short of the systematic study on the effect of modeling establishment. This experiment was conducted to assess the efficacy of ISO on the establishment of CHF and cardiac remodeling model in rats.

\section{Materials and Methods}

\subsection{Animals and Reagents}

Twelve 8-week-old male Wistar rats (weight, 200 230g) used in this study were obtained from Laboratory Animal Center of Sun Yat-sen University (Guangzhou, China). ISO was purchased from Bo Yuan Pharmaceutical Co., Ltd. (Jinan, China). Other reagents include Masson staining kit (PanEra, Guangzhou, China), TRIzol (Invitrogen, CA, USA), All-in-One TM first strand cDNA synthesis kit (GeneCopoeia, Rockville, MD, USA) and All-in-One TM qPCR mix (GeneCopoeia, Rockville, MD, USA). Ultrasound System (GE Vivid 7, New York, USA), Fluorescence microscopy (ZEISS Axio Scope A1, Oberkochen, Germany) and Quantitative PCR instrument (EPPENDORF Mastercycler ep realplex2, Hamburg, Germany) were also used in this study.

2.2. Modeling Method 
Rats were randomly divided into two groups using computer: control group $(n=3)$ and model group $(n=9)$. In the model group, rats were hypodermic injected with ISO $5 \mathrm{mg} / \mathrm{kg} / \mathrm{d}$ from the first day to tenth day. Accordingly, the controls were injected with normal saline. Rats were fasted for 24 hours after the last injection. All of them were executed on the eleventh day.

\subsection{Ultrasonic Cardiogram (UCG)}

Rats were anaesthetized with $2 \%$ pentobarbital sodium $(50 \mathrm{mg} / \mathrm{kg})$. And then, the chest area was shaved for UCG examination. The left ventricular internal diameter at end-systole (LVIDs), left ventricular internal diameter at end-diastole (LVIDd), left ventricular fractional shortening fraction (FS) and left ventricular ejection fraction (EF) were measured. Three consecutive cardiac cycles was saved for each measurement and the average of them was taken for final analysis.

\subsection{Ventricular Mass/Body Mass Ratio (VW/BM)}

Rats were sacrificed after UCG examination. Their hearts were immediately got out and washed with cold normal saline to remove the blood. Cardiac atrium, vessels and adipose tissue were removed and only ventricular tissues were retained. The samples were dried with filter papers and weighed with electronic balance. The VW/BM = ventricular mass $(\mathrm{mg}) /$ body mass $(\mathrm{g})$.

\subsection{Histopathology and Immunohistochemistry}

The ventricular samples were fixed with $10 \%$ formaldehyde solution and embedded with paraffin. Then, the paraffin embedded tissues were sectioned perpendicularly to the longitudinal axis of the heart. The sections were used for HE staining and Masson staining to assess the severity of cardiac fibrosis. In Masson staining, collagen fibers would be stained blue and myocardial tissue would be stained red. Immunohistochemistry of collagen I was also performed. The expression of Collagen I would be observed under microscope. The rest of ventricular tissues were retained in ultra-low temperature freezer $\left(-80^{\circ} \mathrm{C}\right)$.

\subsection{CollagenVolume Fraction (CVF) and Perivascular Collagen Area (PVCA)}

Masson staining sections were also used to calculate the CVF and PVCA. Based on the positive stained area, which scattered between the surviving myocytes and around the blood vessels, collagen volume fraction (CVF) and perivascular collagen area (PVCA) were measured (3). $\mathrm{CVF}=$ collagen area/total area. $\mathrm{PVCA}=$ collagen area around the arterial lumen/arterial lumen area. Six visions under microscope of each sample were randomly chosen and the average of them was taken for analysis.

\subsection{Hydroxyproline (HYP) Concentration}

Put 30 100mg (wet weight) ventricular tissue into $1 \mathrm{ml}$ hydrolytic agent, $95^{\circ} \mathrm{C}$ constant temperature bath for $20 \mathrm{~min}$, adjust the $\mathrm{PH}$ to $6.0 \sim 6.8$. And then, according to the instruction of HYP testing kit (Alkaline method) provided by Nanjing Jiancheng Bioengineering Institute (Nanjing, China), optical density (OD) of each sample was measured. HYP concentration in ventricular tissue $(\mu \mathrm{g} / \mathrm{mg})=($ sample tube OD-blank tube OD) $\times$ standard sample concentration $(5 \mu \mathrm{g} / \mathrm{ml}) \times$ total volume $(10 \mathrm{ml}) /[($ standard tube OD- blank tube OD) $\times$ tissue wet weight $(\mathrm{mg})]$.

\subsection{Real-time Quantitative PCR}

Total RNA was prepared from ventricular tissue by using TRIzol reagent and reverse transcribed to cDNA by using the All-in-One TM first strand cDNA synthesis kit. Two microliter cDNA was amplified by real-time quantitative PCR using the All-in-One TM qPCR mix (SYBR Green method). The primers used in this study were provided by GENEWIZ Co., Ltd. (Suzhou, China). The sequences were shown in Table 1. Each experiment was performed at least three times.

\subsection{Statistical Analysis}

All data were analyzed with IBM SPSS software (Version 20.0). The data were presented as mean \pm standard deviation. Student $t$ test or Wilcoxon test was used according to the result of normality test and homogeneity of variance test. A two-tail $\mathrm{P}<0.05$ was considered to indicate a significant difference.

Table 1. Primers used in the study

\begin{tabular}{cc}
\hline Primer & Sequence \\
\hline TGF- $\beta 1(\mathrm{~F})$ & 5'-TGCTTCAGCTCCACAGAGAA-3' \\
\hline TGF- $\beta 1(\mathrm{R})$ & 5'-TGGTTGTAGAGGGCAAGGAC-3' \\
\hline$\beta$-actin(F) & 5'-AGGGAAATCGTGCGTGACAT-3' \\
\hline$\beta$-actin(R) & 5'-GAACCGCTCATTGCCGATAG-3' \\
\hline
\end{tabular}


Table 2. Comparison of cardiac function and structure

\begin{tabular}{|c|c|c|c|}
\hline & Control & Model & $\mathrm{P}$ \\
\hline LVIDd(mm) & $4.75 \pm 1.19$ & $8.91 \pm 0.22$ & 0.023 \\
\hline LVIDs(mm) & $3.52 \pm 0.79$ & $7.19 \pm 0.32$ & 0.002 \\
\hline $\mathrm{EF}(\%)$ & $57.00 \pm 3.61$ & $44.67 \pm 3.06$ & 0.011 \\
\hline FS (\%) & $25.67 \pm 2.52$ & $19.33 \pm 1.53$ & 0.020 \\
\hline $\mathrm{VM}(\mathrm{mg})$ & $965.40 \pm 86.21$ & $1249.07 \pm 79.51$ & 0.002 \\
\hline $\mathrm{BM}(\mathrm{g})$ & $268.00 \pm 3.00$ & $256.50 \pm 12.74$ & 0.179 \\
\hline $\mathrm{VM} / \mathrm{BM}(\mathrm{mg} / \mathrm{g})$ & $3.60 \pm 0.31$ & $4.88 \pm 0.34$ & 0.020 \\
\hline
\end{tabular}

LVIDd=left ventricular internal diameter at end-diastole; LVIDs=left ventricular internal diameter at end-systole; $\mathrm{EF}=$ ejection fraction; $\mathrm{FS}=$ fractional shortening fraction; $\mathrm{VM}=$ ventricular mass; $\mathrm{BM}=$ body mass

\section{Results}

\subsection{General Data}

During the whole procedure, three rats in model group died. All of them were died within two hours after injection of ISO. No rats died in control group. Compared to the controls, rats in model group became depressive, inactive and shaggy.

\subsection{Comparison of UCG Results}

The LVIDd $(4.75 \pm 1.19 \mathrm{~mm}$ vs. $8.91 \pm 0.22 \mathrm{~mm})$ and LVIDs $(3.52 \pm 0.79 \mathrm{~mm}$ vs. $7.19 \pm 0.32 \mathrm{~mm})$ were increased in model group. Besides, their EF $(57.00 \pm 3.61 \%$ vs. $44.67 \pm 3.06 \%)$ and FS $(25.67 \pm 2.52 \%$ vs. $19.33 \pm 1.53 \%)$ levels decreased significantly, compared to the control group (Table 2).

\subsection{Comparison of Ventricular Mass/Body Mass Ratio}

As shown in Table 2, the ventricular mass (965.40 \pm 86.21 $\mathrm{mg}$ vs. $1249.07 \pm 79.51 \mathrm{mg})$ and $\mathrm{VM} / \mathrm{BM}$ ratio $(3.60 \pm 0.31 \mathrm{vs}$. $4.88 \pm 0.34)$ in model group increased significantly as compared to the controls. No statistical difference of body mass was found between the two groups $(268.00 \pm 3.00 \mathrm{~g}$ vs. $256.50 \pm 12.74 \mathrm{~g})$.

\subsection{Comparison of HE Staining and Masson Staining}

As shown in Figure 1, the myocardial tissues of control group were arranged in order and stained well (A). In the model group, an irregular arrangement of myocardial tissues was found. The tissues were infiltrated with inflammatory cells. The major lesions were located in the subendocardial myocardium area (B). In Masson staining, collagen fibers were stained blue and myocardial tissue were stained red. Compared to the controls (C), the collagen fibers were significantly increased in model group, especially in subendocardial myocardium and perivascular areas (D).

\subsection{Comparison of Immunohistochemistry of Collagen I}

In the immunohistochemistry of collagen I, the positive tissue was stained brown. According to Figure 2, collagen I was found thinly distributed in control group (A). However, the positive area was increased significantly in the model group (B).

\subsection{Comparison of CVF and PVCA}

Compared to the controls, the CVF $(5.65 \pm 0.68 \%$ vs. $27.62 \pm 4.89 \%, \quad \mathrm{P}=0.020)$ and PVCA $(11.22 \pm 3.40 \%$ vs. $28.50 \pm 4.52 \%, \mathrm{p}=0.001)$ were found higher in the model group (Figure 3).

\subsection{Comparison of HYP Concentration}

The HYP concentrations in myocardial tissues were measured. A significant increase of HYP $(0.24 \pm 0.08 \mu \mathrm{g} / \mathrm{mg}$ wet weight vs. $0.62 \pm 0.11 \mu \mathrm{g} / \mathrm{mg}$ wet weight, $\mathrm{P}=0.001$ ) was found in model group as compared to the control group.

\subsection{Comparison of Relative Expression of TGF- $\beta 1$ mRNA}

In this study, $\beta$-actin was used as a reference gene and $\Delta \Delta \mathrm{Ct}$ method was used to analyze the relative expression of TGF- $\beta 1$ mRNA in myocardial tissue. As shown in Figure 4. , compared to the controls, the relative expression of TGF- $\beta 1$ mRNA was found increased significantly in the model group $(\mathrm{P}=0.003)$. 

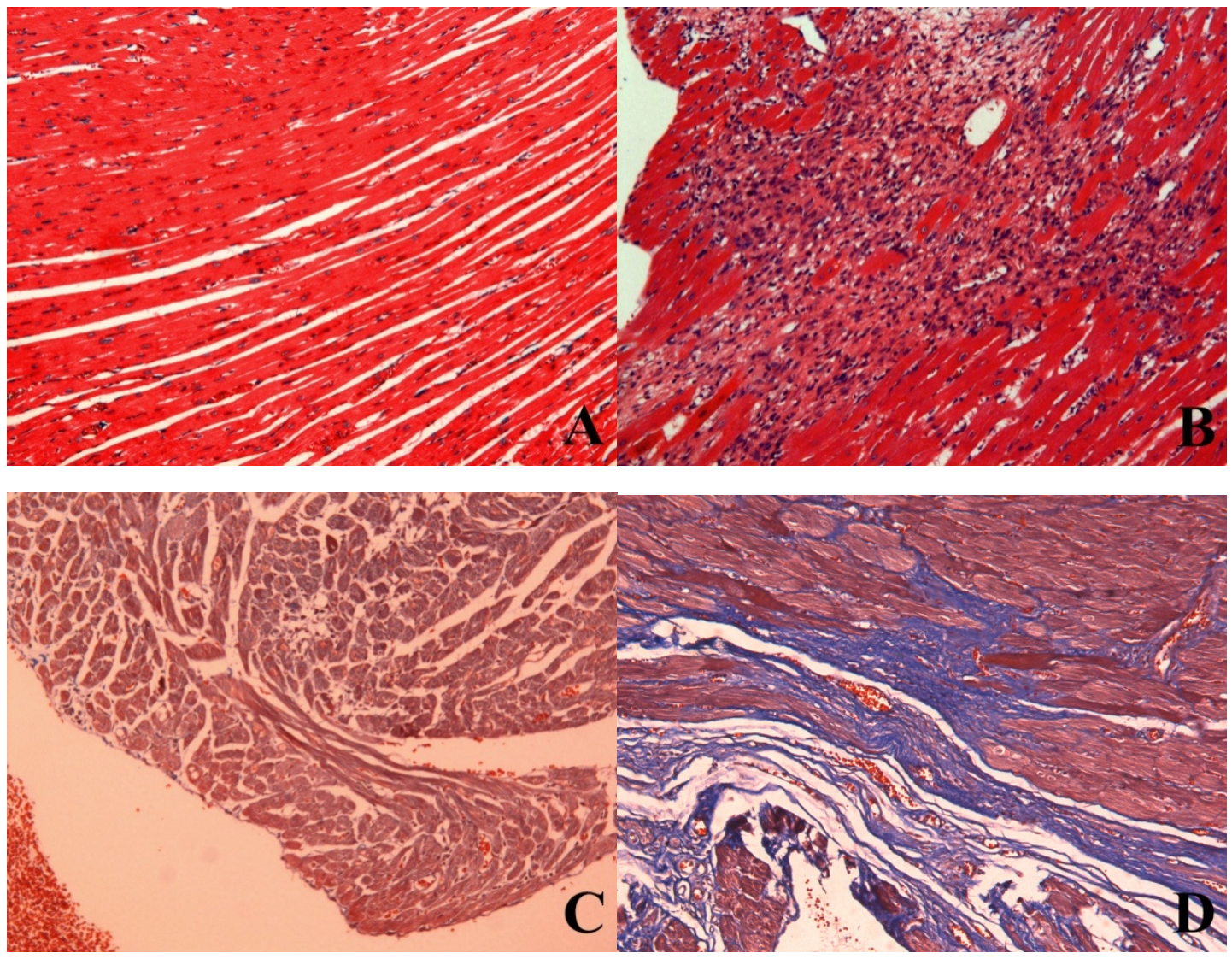

Figure 1. Comparison of histopathology.(A) Myocardial tissues of control group were arranged in order and stained well in control group (HE staining, $100 \times$ ); (B) Myocardial tissues were arranged irregularly and infiltrated with inflammatory cells, especially in the subendocardial myocardium area (HE staining, 100×); (C) Collagen fibers were stained blue and myocardial tissue were stained red. Only few collagen fibers were found in control group (Masson staining, 100×); (D) Collagen fibers were significantly increased in the model group (Masson staining, 100×).

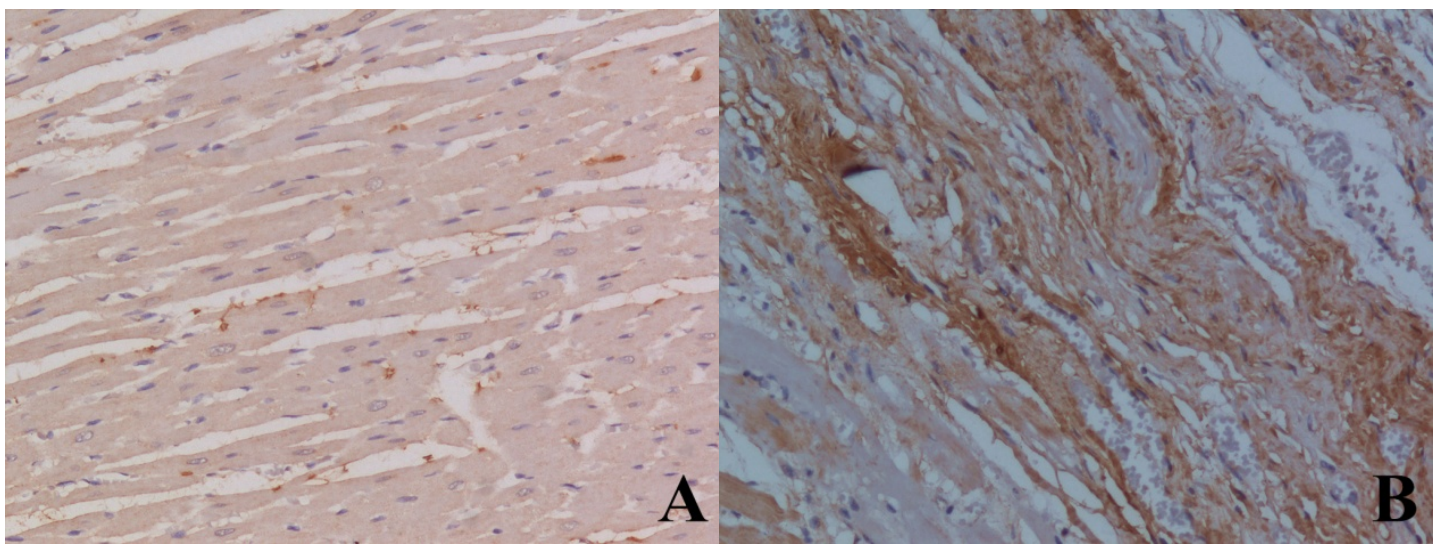

Figure 2. Comparison of Immunohistochemistry of Collagen I . (A) Myocardial tissues were arranged in order and few collagen I was found in control group (Immunohistochemistry, 200×); (B) Collagen I was found increased significantly in the model group (Immunohistochemistry, 200×). 


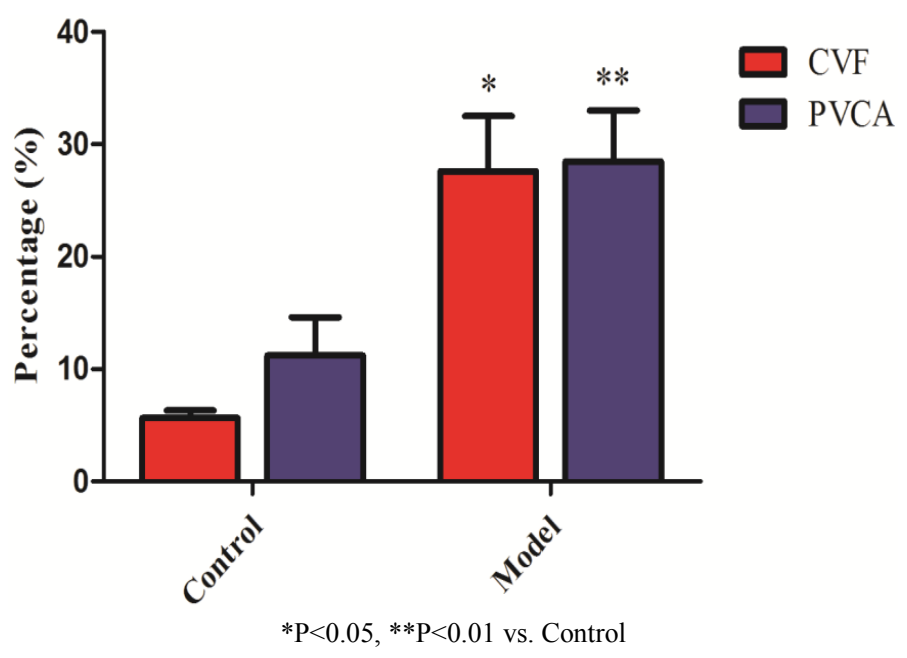

Figure 3. Comparison of CVF and PVCA

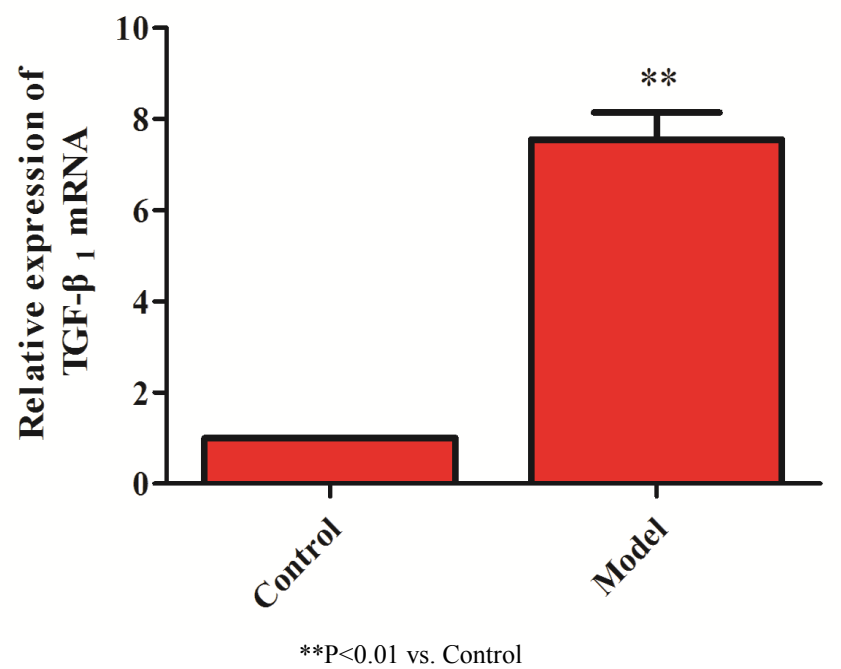

Figure 4. Comparison of Relative Expression of TGF- $\beta 1$ mRNA

\section{Discussion}

Our study established the CHF and cardiac remodeling rat model with hypodermic injection with ISO for 10 days successfully. Compared to the control group, hearts in model group were enlarged and the cardiac function sharply decreased. Histopathology suggested an irregular array of myocardium cells, infiltration of inflammatory cells and increase of collagen $I$ in the model group. The HYP concentration and relative expression of TGF- $\beta 1$ mRNA were significantly increased.

A good animal model is essential for the basic research on CHF and cardiac remodeling [4]. At present, coronary artery ligation or embolization is a relatively common method to set up the animal model of CHF by cutting off blood flow in coronary artery and inducing myocardium cells' necrosis. But this method is more suitable for big animals such as pig and dog, because it has strict requirements for the experimental environment, device and operation skills. Moreover, there is a high risk of death for animals after operation. The rat model of CHF and cardiac remodeling can be also accomplished with drugs, such as adriamycin and catecholamine [5]. ISO is an agitation to $\beta$-receptor of adrenaline, which has a positive function to heart rate and strength of myocardium. Previous studies [4, 6] suggested that large dose of ISO with hypodermic injection could induce necrosis, hyperplasia and myocardial fibrosis. It could also increase the expression of angiotensin II and impair pump function of myocardium in rats. The possible mechanism might be acute diffuse necrosis of myocardium caused by sharp increase of catecholamine. ISO can also cause a relatively lack of blood flow for myocardium. In addition, chronic inflammatory reaction and activation of renin-angiotensin-aldosterone system may also be involved in the progress of CHF and myocardial remodeling [7]. Consequently, compared to coronary artery ligation or embolization, ISO method is preferred by many researchers because of its convenience, lower cost and no need for extra device [8-10].

However, there is no consensus yet for the accurate 
method using ISO in the establishment of CHF model. Firstly, the dose of ISO varies greatly. Previous studies [7, $11-12$ ] suggested that high dose of ISO $(85 \sim 170 \mathrm{mg} / \mathrm{kg} / \mathrm{d})$ could set up CHF model in rats, but acute death rate was up to $55 \% \sim 66 \%$. The mortality was related to the doses. While some other studies suggested that low dose of ISO $(5 \sim 15 \mathrm{mg} / \mathrm{kg} / \mathrm{d})$ could also set up the model and death ratio was around $30 \%$ [9, 13-15]. Secondly, the program steps of ISO injection are different. Garjani and $\mathrm{Li}[13,15]$ used ISO $5 \mathrm{mg} / \mathrm{kg} / \mathrm{d}$ for 10 days to establish CHF model. However, Li [14] used ISO10 $\mathrm{mg} / \mathrm{kg} / \mathrm{d}$ with hypodermic injection for 3 days, and then $5 \mathrm{mg} / \mathrm{kg} / \mathrm{d}$ for another 11days to build the model. All of them reported a successful result. To exam the actual efficiency of ISO in the establishment of CHF and cardiac remodeling rat model, we use ISO $5 \mathrm{mg} / \mathrm{kg} / \mathrm{d}$ by hypodermic injection for 10days with a 24-hour interval between every two injections in this study. The death rate in model group is $33.3 \%$ (3/9). The hearts were enlarged and pump function were decreased apparently. Besides, indicators of cardiac remodeling (HYP and TGF- $\beta 1$ ) were markedly increased. Therefore, the method we use can build the model of CHF and cardiac remodeling successfully and the death rate of rats is low, which is worthy of reference and spread.

However, the study has some limitations. Firstly, the number of rats is relatively low and can cause some bias. Secondly, we didn't set up groups with different doses to make a parallel comparison, so that we can ensure the best dose of ISO. Thirdly, there is no comparison with different combination groups, with which we can find a best medicine combination to minimize the death rate. Future studies are still needed.

In conclusion, ISO can induce CHF and cardiac remodeling rat model successfully with great efficacy and low death rate.

\section{Acknowledgements}

The authors do not report any potential conflict of interest relevant to this article. Equal contributions were made to this work by Dr. Jianting Ke and Dr. Dan Huang. This study was supported by grants from the Natural Science Foundation of Guangdong Province (S2013040014 921), Medical Student Summer Amateur Research Program of Sun Yat-sen University (2014073) and Science Foundation of Zhuhai Science and Technology Bureau (2012031).

\section{REFERENCES}

[1] Yancy C W, Jessup M, Bozkurt B, et al. 2013 ACCF/AHA Guideline for the Management of Heart Failure: Executive
Summary: A Report of the American College of Cardiology Foundation/American Heart Association Task Force on Practice Guidelines. J Am Coll Cardiol, 2013, 62:1495-1539.

[2] Braunwald E. Research advances in heart failure: a compendium. Circ Res, 2013, 113:633-645.

[3] Zhang Z, Zhao P, Li A, et al. Effects of methotrexate on plasma cytokines and cardiac remodeling and function in postmyocarditis rats. Mediators Inflamm, 2009, 2009:38972 0 .

[4] Hasenfuss G. Animal models of human cardiovascular disease, heart failure and hypertrophy. Cardiovasc Res, 1998, 39:60-76.

[5] Liu B, Li M, Luo X, et al. Study on construction method of heart failure experimental animal models. Chinese Journal of Cardiovascular Medicine.2013, 18(3):233-236.

[6] Benjamin I J, Jalil J E, Tan L B, et al. Isoproterenol-induced myocardial fibrosis in relation to myocyte necrosis. Circ Res, 1989, 65:657-670.

[7] Li W, Tian Y, Kong Y, et al. Experimental study of model of isoproterenol-induced heart failure in the rat. Journal of Harbin Medical University. 2003, 37(5):386-388.

[8] Dong R Q, Wang Z F, Zhao C, et al. Toll-Like Receptor 4 Knockout Protects Against Isoproterenol-Induced Cardiac Fibrosis: The Role of Autophagy. J Cardiovasc Pharmacol Ther, 2014. in press (PMID 24950765).

[9] Li M, Jiang Y, Jing W, et al. Quercetin provides greater cardioprotective effect than its glycoside derivative rutin on isoproterenol-induced cardiac fibrosis in the rat. Can $\mathrm{J}$ Physiol Pharmacol,2013,91:951-959.

[10] Feng W, Li W, Liu W, et al. IL-17 induces myocardial fibrosis and enhances RANKL/OPG and MMP/TIMP signaling in isoproterenol-induced heart failure. Exp Mol Pathol,2009,87:212-218.

[11] Suzuki M, Ohte N, Wang Z M, et al. Altered inotropic response of endothelin-1 in cardiomyocytes from rats with isoproterenol-induced cardiomyopathy. Cardiovasc Res, 1998 ,39:589-599.

[12] Teerlink J R, Pfeffer J M, Pfeffer M A. Progressive ventricular remodeling in response to diffuse isoproterenol-i nduced myocardial necrosis in rats. Circ Res, 1994,75:105-11 3.

[13] Garjani A, Andalib S, Biabani S, et al. Combined atorvastatin and coenzyme Q10 improve the left ventricular function in isoproterenol-induced heart failure in rat. Eur J Pharmacol,2 011,666:135-141.

[14] Song L, Jiang W, Hu X, et al. The Antagonistic Effect of P13K-gamma Inhibitor AS605240 on Cardiac Hypertrophy and Cardiac Fibrosis Induced by Isoproterenol In Rats. Journal of Sichuan University (Medical ScienceEdition),201 $1,42: 471-474$.

[15] Li J Z, Peng J, Xiao L, et al. Reversal of isoprenaline-induced cardiac remodeling by rutaecarpine via stimulation of calcitonin gene-related peptide production. Can J Physiol Pharmacol,2010,88:949-959 